\title{
The Novel Oral Anticoagulants
}

\author{
Cristhiam M. Rojas-Hernandez, MD ${ }^{1}$ David A. Garcia, MD² \\ ${ }^{1}$ Division of Hematology/Oncology, University of New Mexico, \\ Albuquerque, New Mexico \\ 2 Division of Hematology, University of Washington, Seattle, \\ Washington

\begin{abstract}
Address for correspondence and reprint requests David Garcia, MD, Division of Hematology, University of Washington, 1100 Fairview Avenue North D5-100, PO Box 19024, Seattle, WA 98109
\end{abstract} \\ (e-mail: davidg99@uw.edu).
}

Semin Thromb Hemost 2013;39:117-126.

\begin{abstract}
Keywords

- anticoagulants

- oral anticoagulants

- apixaban

- rivaroxaban

- dabigatran

After the introduction of warfarin, long-term oral anticoagulation treatment remained unchanged for more than 50 years. Most recently, with the development and approval of new oral anticoagulants, the treatment of medical conditions that require thrombosis prophylaxis and long-term anticoagulation has become more complex. In the case of venous thromboembolism (VTE) prevention after orthopedic surgery, the new oral agents will be less costly than the parenteral alternative. In other settings (such as atrial fibrillation or treatment of acute VTE), the new agents will offer additional convenience at higher cost, but the degree to which they will reduce clinically important events such as thrombosis or bleeding will be limited, especially for patients on optimally controlled warfarin. As the use of the new oral anticoagulants becomes more widespread, it will be important for all clinicians to have a basic understanding of their pharmacology, advantages, and limitations. Although the need to measure or reverse the effect of these drugs will arise infrequently, clinicians-especially hematologists-will desire evidence-based recommendations about how to manage such scenarios, which will require research studies.
\end{abstract}

Three new oral anticoagulant drugs (apixaban, rivaroxaban, and dabigatran) have become available for prophylaxis and treatment of acute venous thromboembolism (VTE), and, since 2010, also for prevention of stroke and systemic embolism in patients with nonvalvular atrial fibrillation (AF). This represents a significant change, because since the introduction of warfarin half a century ago, there had been no significant changes in drugs available for use in long-term anticoagulation. The favorable features of the new oral agents (i.e., less complex drug-to-drug interaction profiles, the lack of need for routine monitoring) make them an attractive option for managing anticoagulation in the inpatient and outpatient settings. On the contrary, the new agents have important limitations (e.g., contraindication in severe renal insufficiency, lack of an antidote in case of bleeding, and higher cost than warfarin) that complicate clinical decisions about when and how to use them. This review describes the landmark clinical trial data as well as practical considerations that are relevant to treating patients with the three new oral agents (apixaban, dabigatran, and rivaroxaban) that are now in advanced stages of clinical development.

published online February 6, 2013
Issue Theme Expert Approaches to Common Bleeding and Thrombotic Problems; Guest Editors, Catherine P. M. Hayward, MD, PhD, FRCPC, and Kathryn E. Webert, MD, MSc, FRCPC.

\section{Pharmacology}

All drug dosage recommendations in this article are for North America, unless otherwise stated. The doses approved may differ in other regions.

\section{Apixaban}

Apixaban is a reversible, direct, and highly selective active site inhibitor of factor Xa (FXa). It does not require antithrombin for its anticoagulant activity. Apixaban inhibits free and clotbound FXa, and prothrombinase activity. Apixaban has no direct effects on platelet aggregation, but indirectly inhibits tissue factor-induced platelet aggregation in vitro, presumably by inhibiting the production of thrombin. ${ }^{1,2}$

Apixaban is produced as a $2.5 \mathrm{mg}$ film-coated tablet. The bioavailability of apixaban is approximately $50 \%$; it is rapidly absorbed, not affected by food, with maximum concentrations appearing 3 to 4 hours after tablet intake and has a halflife of approximately 12 hours. ${ }^{3}$ Apixaban is metabolized mainly via the cytochrome P450 (CYP) enzyme 3A4/5. ${ }^{3}$ Renal

Copyright (c) 2013 by Thieme Medical Publishers, Inc., 333 Seventh Avenue, New York, NY 10001, USA. Tel: +1(212) 584-4662.
DOI http://dx.doi.org/ $10.1055 / \mathrm{s}-0032-1333536$. ISSN 0094-6176. 
excretion of apixaban accounts for approximately $27 \%$ of total clearance with additional contributions from biliary and direct intestinal excretion. ${ }^{3}$

The recommended apixaban doses for most patients are $5 \mathrm{mg}$ twice daily for stroke prevention in AF and $2.5 \mathrm{mg}$ twice daily for the prevention of VTE after orthopedic surgery. No dose adjustment is necessary in patients with mild renal impairment. There is no clinical experience in patients with creatinine clearance $(\mathrm{CrCl})$ less than $15 \mathrm{~mL} / \mathrm{min}$, or in patients undergoing dialysis. ${ }^{4}$ No dose adjustment is required in patients with mild or moderate hepatic impairment (Child Pugh A or $B$ ), but apixaban is contraindicated in patients with hepatic disease associated with coagulopathy. ${ }^{4}$

No additional adjustment is necessary by body weight or age. There are no data available from the use of apixaban in pregnant women, and apixaban is not recommended during pregnancy. It is unknown whether apixaban or its metabolites are excreted in human milk. Available data in animals have shown excretion of apixaban in milk. ${ }^{5}$

\section{Rivaroxaban}

Rivaroxaban is an oral FXa inhibitor that selectively blocks the active site of FXa and, like apixaban, does not require antithrombin for its activity.

Rivaroxaban is manufactured as tablets: 10, 15, and $20 \mathrm{mg}$. The absolute bioavailability is more than $50 \%$ and it is dosedependent; at $10 \mathrm{mg}$ dose, it is estimated to be 80 to $100 \%$ bioavailable. The bioavailability of rivaroxaban is not decreased by food and it is not affected by drugs that alter gastric $\mathrm{pH}^{6,7}$

The maximum concentrations of rivaroxaban appear 2 to 4 hours after oral intake and the elimination half-life of rivaroxaban is 5 to 9 hours. Rivaroxaban is metabolized mainly via oxidative degradation in the liver. ${ }^{8}$ In humans, CYP3A4 and CYP2J2 are the two enzymes responsible for its oxidative metabolism. ${ }^{8}$ Inhibitors and inducers of these CYP enzymes can result in changes in rivaroxaban exposure.

Approximately $40 \%$ of the unchanged drug is excreted into the urine due to elimination by active tubular secretion. ${ }^{6}$ Rivaroxaban is a moderate substrate of the efflux transporter P-glycoprotein (P-gp). Drugs that inhibit both the CYP3A4 enzymes and the P-gp include ketoconazole, ritonavir, clarithromycin, fluconazole, and erythromycin. ${ }^{9}$ The concomitant use of rivaroxaban and these medications could increase blood levels of rivaroxaban and bleeding risk. ${ }^{9}$

The recommended postoperative thromboprophylaxis (knee and hip replacement) is $10 \mathrm{mg}$ once daily. The therapeutic dose ranges between 15 and $20 \mathrm{mg}$ once daily, and needs adjustment based on the estimated $\mathrm{CrCl}$. For patients with $\mathrm{CrCl}>50 \mathrm{~mL} / \mathrm{min}, 20 \mathrm{mg}$ is the recommended daily dose; with $\mathrm{CrCl}<50 \mathrm{~mL} / \mathrm{min}, 15 \mathrm{mg}$ is the recommended daily dose. There is no clinical experience in patients with $\mathrm{CrCl}<30 \mathrm{~mL} / \mathrm{min}^{7}$ For the first 21 days of treatment for acute DVT or PE, rivaroxaban is given at a dose of $15 \mathrm{mg}$ orally twice daily.

Rivaroxaban has not been studied in patients with severe hepatic impairment (Child Pugh C). For patients with moder- ate hepatic impairment (Child Pugh B), the mean rivaroxaban exposure is increased by 2.3 -fold. ${ }^{7}$

The safety and effectiveness of rivaroxaban during labor and delivery have not been studied in clinical trials; it is not known if rivaroxaban is excreted in human milk.

\section{Dabigatran Etexilate}

Dabigatran etexilate is a pro-drug that is converted by tissue esterases to dabigatran, a competitive, direct thrombin inhibitor. ${ }^{10}$ There is an evidence from in vitro experiments that both free and clot-bound thrombin are inhibited by dabigatran. $^{2}$ Also, there is evidence of inhibition of tissue factorinduced platelet aggregation by this medication. ${ }^{2}$

Dabigatran is available in the United States as 75 and $150 \mathrm{mg}$ capsules. In many other jurisdictions, dabigatran is available as 110 and $150 \mathrm{mg}$ capsules. The absolute bioavailability of dabigatran following oral administration is approximately 3 to $7 \%$. The absorption is influenced by the intestinal efflux transporter P-gp. The maximum concentration occurs at 1 hour postadministration in the fasted state; and can be delayed by approximately 2 hours if administered with meals, but the presence of food does not change the ultimate bioavailability of dabigatran. The half-life of dabigatran etexilate is 12 to 17 hours. ${ }^{10}$

Dabigatran is not a substrate, inhibitor, or inducer of CYP450 enzymes and is eliminated primarily in the urine. ${ }^{11}$ In adults with moderate hepatic impairment (Child Pugh B), there is no evidence of a consistent change in exposure or pharmacodynamics. ${ }^{12}$

The recommended dose of dabigatran is $150 \mathrm{mg}$ taken orally, twice daily; a dose reduction to $75 \mathrm{mg}$ twice daily is indicated (in the United States) for patients with $\mathrm{CrCl}$ $<30 \mathrm{~mL} / \mathrm{min}^{12}$ There is no clinical trial experience in patients with a $\mathrm{CrCl}<30 \mathrm{~mL} / \mathrm{min}$ (the $75 \mathrm{mg}$ dose was approved based on pharmacokinetic modeling) and the manufacturer recommends that dabigatran should not be used in patients with a $\mathrm{CrCl}<15 \mathrm{~mL} / \mathrm{min}$ or in patients who require renal replacement therapy.

The concomitant use of dabigatran etexilate with P-gp inducers (e.g., rifampicin) reduces exposure to dabigatran and should generally be avoided ${ }^{13}$; although concomitant P-gp inhibitors may increase exposure to dabigatran, ${ }^{14}$ the prescribing information approved by the U.S. Food and Drug Administration (FDA) does not require a dose adjustments in such situations. There are no well-controlled studies in pregnant women, and it is not known whether dabigatran is excreted in human milk.

\section{Clinical Trial Evidence}

\section{Apixaban}

Available phase III clinical trial data support the use of apixaban for VTE prophylaxis after orthopedic surgery and for cardioembolic prophylaxis in AF (-Tables 1-3). Phase II studies indicate that apixaban may be a safe and effective option for the treatment of VTE but data from pivotal phase III trials are pending. 


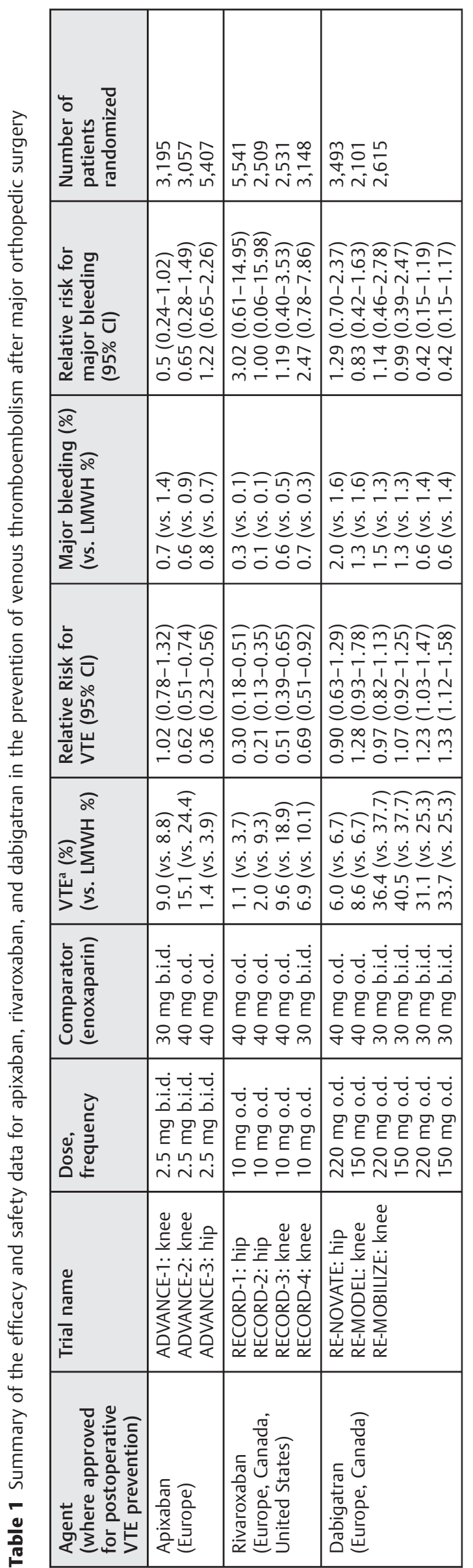

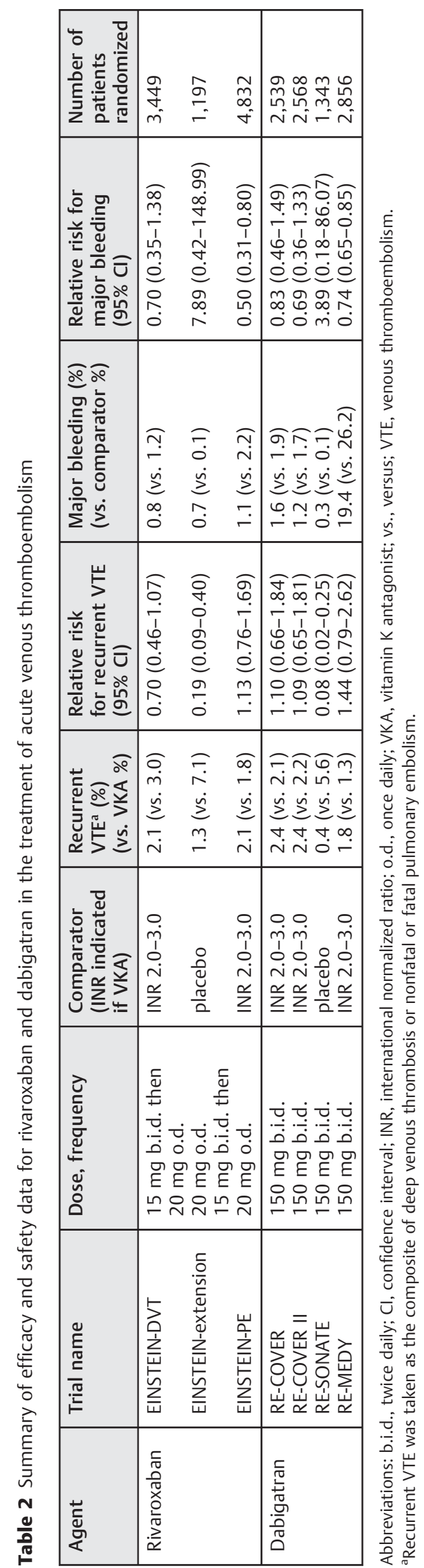




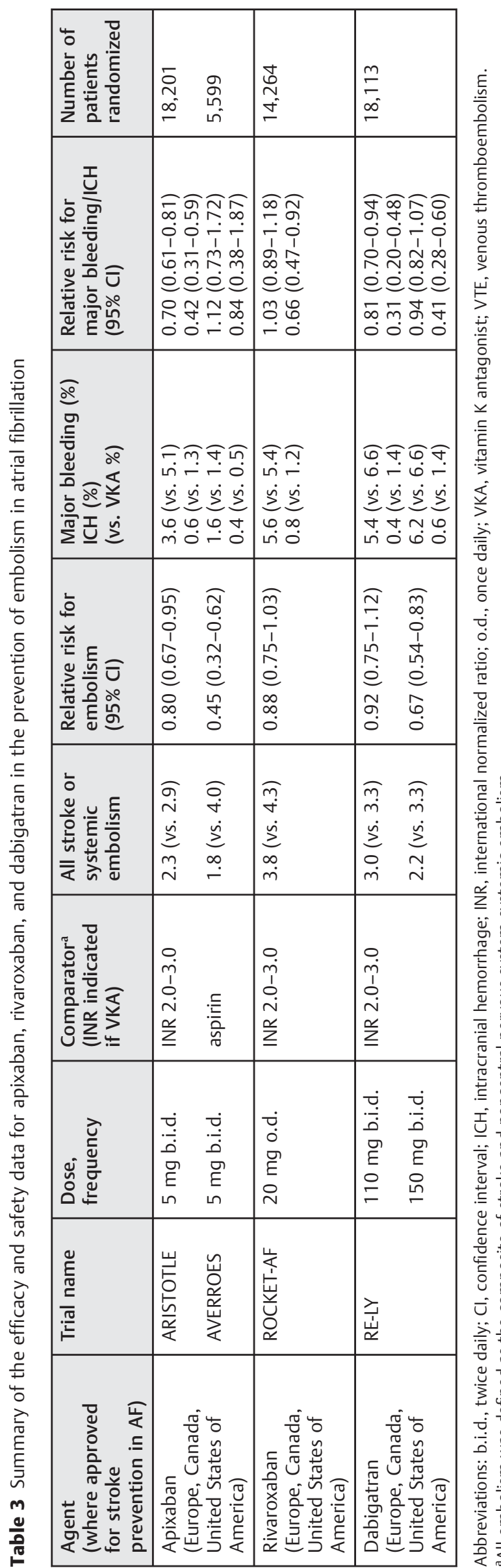

Taken together, the clinical trial data for apixaban as prophylaxis against VTE after orthopedic surgery, demonstrate that apixaban is as effective as low-molecular-weight heparin (LMWH), with a trend toward lower rates of major hemorrhagic complications ${ }^{15-17}$ (-Table $\mathbf{1}$ ).

For thromboprophylaxis in nonsurgical patients, the ADOPT trial randomly assigned acutely ill patients who had congestive heart failure, respiratory failure, or other medical disorders and at least one additional risk factor for deep vein thrombosis (DVT) to 6 days of LMWH or 30 days of apixaban. ${ }^{18}$ All included patients were hospitalized with an expected stay of at least 3 days. The primary efficacy outcome was the 30-day composite of death related to pulmonary embolism (PE), symptomatic DVT, or asymptomatic proximal-leg DVT, as detected with the use of systematic bilateral compression ultrasonography on day 30. Apixaban was not superior to enoxaparin for the primary efficacy outcome; however, the patients randomized to apixaban had a trend toward higher rate of major bleeding during treatment (relative risk [RR], 2.53; 95\% confidence interval [CI], 0.98 to 6.50$).^{18}$

In the VTE treatment setting, a phase II study evaluated apixaban versus LMWH followed by a vitamin $\mathrm{K}$ antagonist (VKA) in patients with symptomatic DVT. Although not powered to yield definitive conclusions, the results of this trial ${ }^{19}$ were sufficiently promising that two additional phase III trials (AMPLIFY, AMPLIFY-EXT) evaluating the efficacy of apixaban in this setting are now near completion.

Apixaban has been evaluated for the treatment of recent acute coronary syndrome (ACS) in the APPRAISE trials. Both of these studies were terminated prematurely because of an increased rate of major bleeding events in the apixaban arm in the absence of a clinically significant reduction in recurrent ischemic events. Nearly all patients in these trials received concurrent standard antiplatelet therapy..$^{20,21}$

For the prevention of stroke in patients with AF, the ARISTOTLE trial showed that apixaban was superior to warfarin for efficacy (all stroke plus systemic embolism), safety (major bleeding), and all-cause death ${ }^{22}$ ( - Table 3 ). Moreover, the AVERROES trial (aspirin vs. apixaban in patients who were not suitable for warfarin) was stopped early because of a lower rate of stroke in the apixaban-treated patients with AF. Although no difference in clinically significant bleeding was observed, the safety results must be interpreted with caution because the number of bleeding events was $\operatorname{low}^{23}$ ( - Table 3 ).

\section{Rivaroxaban}

This direct FXa inhibitor has supporting clinical trial data for its use in VTE prophylaxis and treatment, and cardioembolic prevention in $\mathrm{AF}$ ( $\mathbf{-}$ Tables 1-3). Robust clinical trial evidence indicates that rivaroxaban is a very effective medication for VTE prevention following joint replacement surgery. Four separate trials, involving more than 13,000 patients in total, demonstrated that rivaroxaban-treated patients had a lower rate of total (symptomatic and asymptomatic) VTE than did patients who received enoxaparin. ${ }^{24-27} \mathrm{~A}$ trend toward excess bleeding in the rivaroxaban-treated patients has been noted; however, a prespecified pooled analysis highlights the 
very low overall rate of serious hemorrhagic events ${ }^{28}$ (-Table 1).

The MAGELLAN study, a multicenter randomized controlled trial (RCT), evaluated the efficacy of rivaroxaban for 35 days versus enoxaparin for 10 days in patients hospitalized for various acute medical illnesses with risk factors for VTE. The primary efficacy outcome was the composite of asymptomatic proximal DVT, symptomatic DVT, symptomatic nonfatal PE, and VTE-related death. The primary safety outcome was the composite of major bleeding and clinically relevant nonmajor bleeding. ${ }^{29}$ The modified intent-to-treat analysis showed comparable rates for the primary efficacy outcome (3.0\% in the rivaroxaban group vs. $3.1 \%$ in the enoxaparin group; $\mathrm{RR}, 0.99 ; 95 \% \mathrm{CI}, 0.75$ to $1.30 ; p=0.95$ ), but the safety outcome favored enoxaparin, with a higher rate of bleeding in the rivaroxaban group ( 4.1 vs. $1.7 \%$; RR, $2.5 ; 95 \% \mathrm{CI}, 1.85$ to 3.25; $p<0.0001) .^{30}$

In the DVT treatment setting, the EINSTEIN-DVT study and the EINSTEIN-extension trial ${ }^{31}$ have demonstrated that the efficacy of rivaroxaban for acute treatment and secondary prevention of DVT is comparable to that provided by traditional therapy with LMWH followed by long-term VKA. Furthermore, recent data from the EINSTEIN-PE trial that rivaroxaban is noninferior to standard therapy in patients with symptomatic PE with or without concurrent DVT $^{32}$ (-Table 2).

The ATLAS ACS-TIMI 51 study assessed the efficacy of rivaroxaban in the treatment of ACS. $^{33}$ This randomized, double-blind, placebo-controlled trial compared one of two twice-daily doses ( 2.5 or $5 \mathrm{mg}$ ) of rivaroxaban to placebo. All patients received other standard therapy for ACS (e.g., antiplatelet medications) at the discretion of the treating physician. The primary efficacy end point, a composite of death due to cardiovascular causes, myocardial infarction, or stroke, occurred less frequently in the rivaroxaban-treated patients for both $2.5 \mathrm{mg}$ (hazard ratio [HR], $0.84 ; 95 \% \mathrm{Cl}, 0.72$ to 0.97 ; $p=0.02$ ) and $5 \mathrm{mg}$ doses (HR, 0.85 ; $95 \% \mathrm{Cl}, 0.73$ to 0.98 ; $p=0.03$ ). Moreover, the twice-daily $2.5 \mathrm{mg}$ dose of rivaroxaban reduced the rates of death from cardiovascular causes (2.7 vs. $4.1 \%$; $\mathrm{HR}, 0.66 ; 95 \% \mathrm{Cl}, 0.51$ to $0.86 ; p=0.002$ ) and death from any cause ( 2.9 vs. $4.5 \%$; $\mathrm{HR}, 0.68 ; 95 \% \mathrm{CI}, 0.53$ to $0.87 ; p=0.002$ ); this survival benefit was not seen with the twice-daily $5 \mathrm{mg}$ dose, perhaps because it caused more major bleeding.

The risk of clinically significant bleeding with rivaroxaban increased among patients with ACS in a dose-dependent manner. ${ }^{33}$ As compared with placebo, rivaroxaban increased the rates of major bleeding not related to coronary artery bypass grafting ( 2.1 vs. $0.6 \%$; HR, 3.96; $95 \% \mathrm{CI}, 2.46$ to 6.38 ; $p<0.001)$ and the rates of intracranial hemorrhage (ICH) $(0.6$ vs. $0.2 \% ; p=0.009$ ). The twice-daily $2.5 \mathrm{mg}$ dose resulted in fewer fatal bleeding events than the twice-daily $5 \mathrm{mg}$ dose ( 0.1 vs. $0.4 \% ; p=0.04$ ). The ACS population that will derive a clear-cut net clinical benefit from adding rivaroxaban to other antithrombotic therapies has yet to be defined.

The ROCKET AF trial ${ }^{34}$ showed that rivaroxaban was not inferior to warfarin for the prevention of stroke or systemic embolism in patients with high-risk nonvalvular AF; rivar- oxaban-treated patients exhibited a statistically significant reduction in the risk of intracranial bleeding ( - Table 3 ).

\section{Dabigatran Etexilate}

The RE-NOVATE, the RE-MODEL, and the RE-MOBILIZE trials have explored the efficacy of dabigatran as VTE prophylaxis following elective hip, elective knee replacement surgery, respectively. ${ }^{35-37}$ Taken together, the results of these trials suggest that dabigatran is an effective and safe VTE prevention option following total joint replacement; however, the RE-MOBILIZE trial, which demonstrated superiority for twice-daily enoxaparin over dabigatran, suggests that the optimal VTE prophylaxis dose of this drug is not yet established $^{35-37}$ (- Table $\left.\mathbf{1}\right)$.

For treatment of acute VTE, dabigatran has been compared with standard therapy in the RE-COVER and RE-COVER II trials. ${ }^{38,39}$ The primary outcome in both studies was the 6 month incidence of recurrent symptomatic VTE and related deaths ( $\mathbf{- T a b l e ~} \mathbf{2}$ ). In both trials, the patients assigned to dabigatran were treated with LMWH or unfractionated heparin for 5 to 11 days prior to the initiation of dabigatran. In the control arm of both studies, parenteral therapy was overlapped with VKA treatment until a therapeutic international normalized ratio (INR) was achieved. These studies showed that dabigatran is as effective as LMWH followed by VKA for secondary VTE prevention, without an increase in the bleeding rate when compared to more traditional pharmacologic measures. ${ }^{38,40}$ Furthermore, the data from the RE-SONATE and RE-MEDY studies have demonstrated the safety and efficacy of continuing dabigatran for at least 6 more months beyond the initial treatment for $\mathrm{VTE}^{39,41}$ (-Table 2). There was a slightly higher rate of treatment discontinuation in the dabigatran group related to nonhemorrhagic adverse events, mainly dyspeptic symptoms. ${ }^{38}$ In the RE-MEDY study, a higher rate of ACS was observed in the dabigatran arm. The extent to which this difference may be explained by an imbalance in the baseline diabetes and hypertension between the groups assigned to the different treatment arms is not known. ${ }^{41}$

In patients with recent ACS, dabigatran has been evaluated for the prevention of recurrent cardiovascular ischemic events in a placebo-controlled multicenter phase II dose escalation RCT. ${ }^{42}$ The overall rate of the efficacy outcome was low, with minor differences between the treatment groups. The rate of clinically relevant bleeding was dosedependent, with $93 \%$ of the events occurring within the first 3 days of therapy; most of the patients who experienced bleeding were receiving concomitant dual antiplatelet therapy (aspirin and clopidogrel or ticlopidine) as part of the standard medical management of ACS at randomization. ${ }^{42}$ Even at the lowest dose of dabigatran, the $1.3 \%$ absolute increase in the 6-month bleeding rate could not be justified by any off-setting benefit from this drug.

For stroke prevention in patients with AF, the RE-LY trial ${ }^{43}$ evaluated patients with nonvalvular AF with an increased risk of stroke, who were randomized to one of two fixed doses of dabigatran, or open-label use of warfarin ( - Table 3 ). Concomitant use of antiplatelet agents was discouraged but low- 
dose aspirin was permitted in this study, and the median duration of follow-up was 2 years. Dabigatran $150 \mathrm{mg}$ twice daily was superior to warfarin, and dabigatran $110 \mathrm{mg}$ twice daily was noninferior to warfarin for the prevention of stroke or systemic embolism.

The rates of major and intracranial bleeding were higher with warfarin than with either the $110 \mathrm{mg}$ dose of dabigatran or the $150 \mathrm{mg}$ dose of dabigatran (-Table 3). Not surprisingly, when compared with the $110 \mathrm{mg}$ twice-daily dose, the $150 \mathrm{mg}$ twice-daily dose of dabigatran was associated with a higher rate of overall major bleeding (3.31\% per year vs. $2.87 \%$ per year; RR, $1.16 ; 95 \% \mathrm{CI}, 1.00$ to $1.34 ; p=0.04$ ), mainly from the gastrointestinal tract, and a nonsignificant trend toward more intracranial bleeding $(0.19 \%$ per year vs. $0.10 \%$ per year; RR, $1.90 ; 95 \% \mathrm{CI}, 0.94$ to $3.81 ; p=0.07$ ). In a subgroup analysis, the treatment effect of dabigatran appeared to change based on age; dabigatran-associated major bleeding was more common than warfarin-associated bleeding in patients older than 75 years. ${ }^{44}$ Dyspeptic symptoms were significantly more common with dabigatran than with warfarin (5.8\% in the warfarin group; 11.8 and $11.3 \%$ in the 110 and $150 \mathrm{mg}$ dabigatran groups, respectively; $p<0.001$ for both comparisons).
Although there was an almost statistically significant trend toward a higher rate of myocardial infarction with dabigatran at both doses versus warfarin, a strong trend toward less all-cause mortality was observed in the $150 \mathrm{mg}$ twice-daily dabigatran group compared with the warfarin group.

The FDA has not approved the $110 \mathrm{mg}$ dose of dabigatran because their analyses failed to identify a population where the "net benefit" would be greater at the lower dose. ${ }^{45}$ In other jurisdictions such as Europe and Canada, the $110 \mathrm{mg}$ dose has been approved by the applicable regulatory agencies.

\section{Clinical Application}

The novel oral anticoagulants have a role in the management of acute VTE, stroke prophylaxis in patients with AF, and in the primary prevention of VTE after joint replacement surgery. An effectiveness overview and meta-analysis of the available data has been recently published for the novel anticoagulants in the management of VTE and AF. ${ }^{46}$ For these indications, the target-specific oral agents have demonstrated efficacy and safety that are comparable to more traditional alternatives, such as LMWH or warfarin (-Figs. 1 and $\mathbf{2}$ ). ${ }^{46}$ In the case of VTE prevention after orthopedic surgery, the new
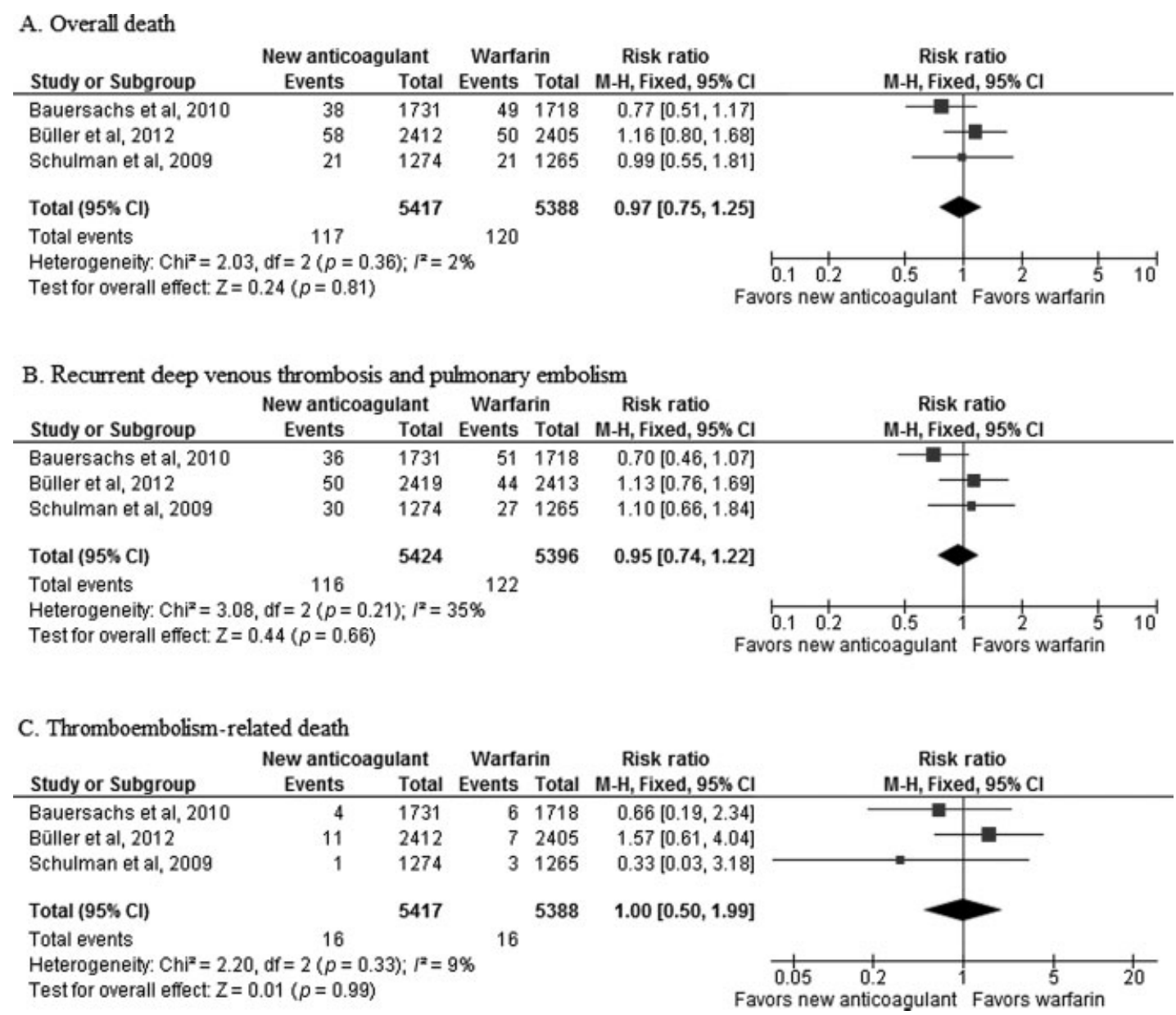

Fig. 1 Comparison of outcomes for the treatment of venous thromboembolism with the new oral anticoagulants compared with warfarin. Panels compare the key outcomes of venous thromboembolism including (A) overall death, (B) recurrent deep venous thrombosis and pulmonary embolism, and (C) thromboembolism-related death. 
A. Overall death

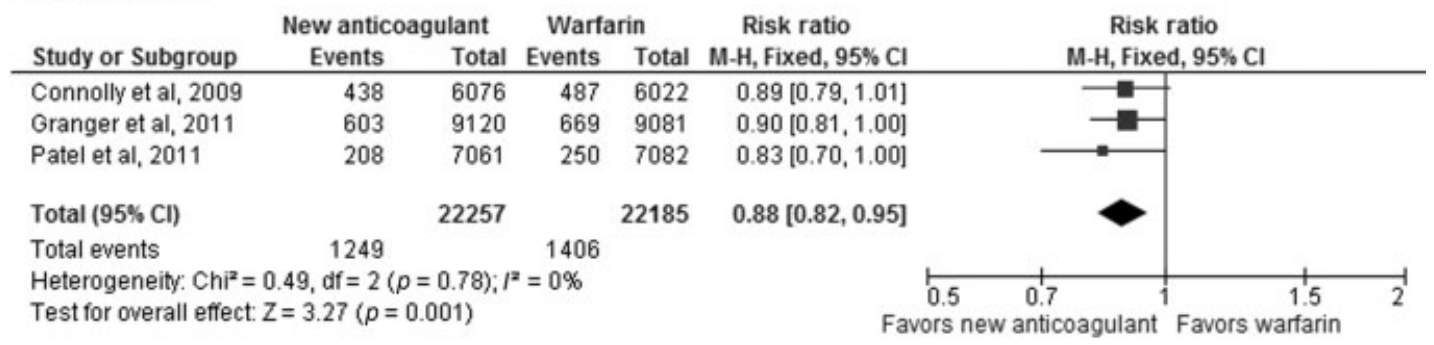

B. Hemorrhagic stroke

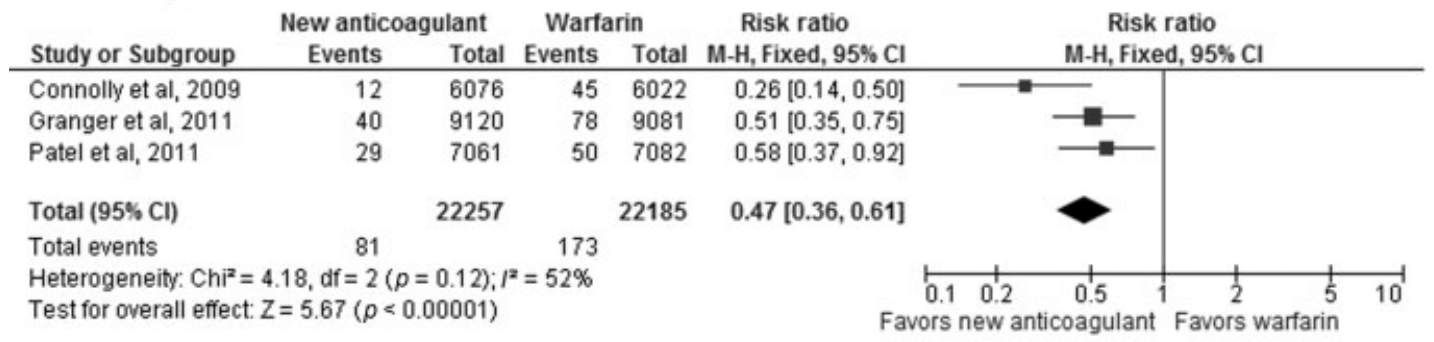

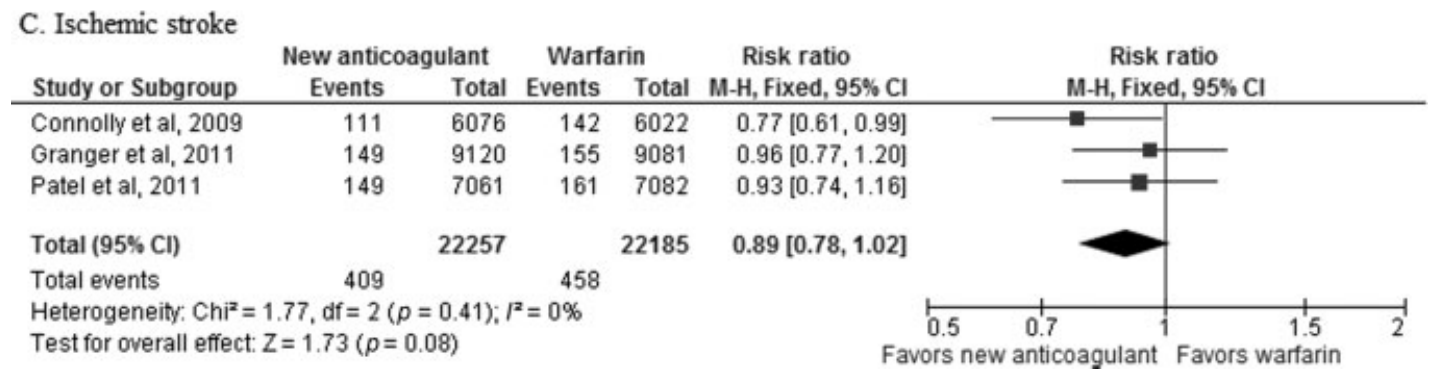

Fig. 2 Comparison of outcomes of treatment to prevent cardioembolism from atrial fibrillation with the new oral anticoagulants compared with warfarin. Panels show outcomes of (A) overall death, (B) hemorrhagic stroke, and (C) ischemic stroke.

agents will be less costly overall than the parenteral alternative (LMWH or fondaparinux) currently used in many institutions. This cost savings, in light of the oral route of administration and the favorable clinical data, make the novel drugs especially attractive for this indication. In other settings (such as AF or VTE), the new agents will offer additional convenience at higher cost, but the degree to which they reduce clinically important events such as thrombosis or bleeding will be limited, especially for patients on optimally controlled warfarin. We suggest that, pending further evidence, the new oral agents be avoided or used with caution in patients who have highly pro-thrombotic conditions (e.g., patients with cancer-associated VTE or patients with bona fide antiphospholipid syndrome). Patients who, despite adhering to recommendations from their provider, have INR values frequently outside the therapeutic range while on warfarin may stand to gain the most from these newer drugs. That notwithstanding, warfarin-treated patients with low time-in-therapeutic range because of poor compliance are probably not good candidates for the novel oral agents. The short half-lives of the newer medicines and the infrequent need for outpatient follow-up would make drugs such as apixaban, dabigatran, and rivaroxaban especially problematic for a patient who did not follow instructions on warfarin. Furthermore, the inability to easily measure the anticoagu- lant effect of the novel agents and the lack of an available antidote or evidence-based reversal strategy are disadvantages to consider.

As patients with severe renal failure or hemodialysis were excluded from the clinical trials on the new oral anticoagulants, they should not be started on these medications. Although the U.S. prescribing information for dabigatran includes a dose recommendation for patients with $\mathrm{CrCl}$ between 15 and $30 \mathrm{~mL} / \mathrm{min}$, the dose ( $75 \mathrm{mg}$ twice daily) is based on pharmacokinetic modeling and has not been tested in a large clinical trial. Finally, warfarin and other VKA remain the only anticoagulant options for patients with mechanical heart valve replacement because the efficacy of the novel agents has not been evaluated in this setting.

\section{Measuring the Anticoagulant Effect and Managing Hemorrhagic Complications}

\section{Apixaban}

As a result of FXa inhibition, apixaban can prolong prothrombin time (PT), and the activated partial thromboplastin time (APTT). However, changes observed in these clotting tests at the expected therapeutic dose are often very small and vary significantly depending, in part, on the reagent used in the assay. A properly calibrated anti-FXa chromogenic assay (using 
Table 4 Oral anticoagulants under clinical development

\begin{tabular}{|l|l|l|}
\hline Drug & Mechanism of action & Phase of development \\
\hline TTP889 & FIXa inhibitor & Phase II trials in VTE prophylaxis for hip replacement \\
\hline Betrixaban & FXa inhibitor & Phase II trials in VTE prophylaxis for knee replacement, AF \\
\hline Darexaban & FXa inhibitor & $\begin{array}{l}\text { Phase II/III trials in VTE prophylaxis for hip and knee replacement, } \\
\text { phase II for ACS and AF }\end{array}$ \\
\hline Edoxaban & $\begin{array}{l}\text { Phase II/III trials in VTE prophylaxis for hip and knee replacement, } \\
\text { phase II/III for AF }\end{array}$ \\
\hline Eribaxaban & FXa inhibitor & Phase II trials in VTE prophylaxis for hip and knee replacement \\
\hline Letaxaban & FXa inhibitor & $\begin{array}{l}\text { Phase II trials in VTE prophylaxis for knee replacement, and } \\
\text { treatment in ACS }\end{array}$ \\
\hline LY517717 & FXa inhibitor & Phase II trial in VTE prophylaxis for knee replacement \\
\hline AZD0837 & Direct thrombin inhibitor & Phase II trials in cardioembolic prophylaxis for AF \\
\hline
\end{tabular}

Abbreviations: ACS, acute coronary syndromes; AF, atrial fibrillation; FIXa, activated factor IX; FXa, activated factor X; VTE, venous thromboembolism. Adapted from Ahrens et al (2012). ${ }^{54}$

an apixaban standard) can exhibit a close direct linear relationship with apixaban plasma concentration. ${ }^{3}$

\section{Rivaroxaban}

Rivaroxaban causes a dose-dependent prolongation of the PT with most reagents. ${ }^{6}$ A normal PT measurement can reassure the clinician that little or no rivaroxaban is present, especially if neoplastin is used as the reagent. ${ }^{6}$ As with apixaban, the anticoagulant effect of rivaroxaban can be assessed with a properly calibrated chromogenic anti-FXa activity measurement (using a rivaroxaban standard). ${ }^{47}$

\section{Dabigatran}

Dabigatran etexilate prolongs the APTT and the thrombin time (TT). The PT/INR is relatively insensitive to dabigatran, but the APTT can, if it is entirely normal, suggest that very little anticoagulant effect is present. ${ }^{48}$ The degree of anticoagulant activity can also be assessed by the ecarin clotting time (ECT). This test is a more specific measure of the effect of dabigatran than the APTT. ${ }^{10}$ The TT assay provides a linear response to dabigatran ${ }^{10,49}$; modified TT assays to measure dabigatran levels are available in some countries. The HEMOCLOT (HYPHEN BioMed, France) direct thrombin inhibitor assay is a sensitive assay that involves highly purified human thrombin to initiate coagulation and has demonstrated a good correlation with plasma levels of dabigatran. ${ }^{50}$ The HEMOCLOT assay, which is already registered in the European Union and Canada, could be useful to evaluate for excessive dabigatran activity in patients presenting with bleeding, or in patients undergoing elective surgery. ${ }^{50}$

The management of clinically significant hemorrhagic complications should include supportive measures: immediately begin resuscitation (e.g., red blood cell transfusions, if required), discontinue the anticoagulant medication, and consider investigations to identify and treat the local source of bleeding. Unfortunately, there is no specific antidote for acute reversal of the effect of these agents. Invasive procedures (e.g., endoscopy) are typically avoided until the anticoagulant effect has worn off.
On the basis of preclinical data, such as animal bleeding models and in vitro coagulation testing, some authors have recommended considering high-dose prothrombin complex concentrates or recombinant factor VIla in cases of severe, life-threatening bleeding in patients receiving a novel oral anticoagulant. While the rationale for and possible merits of these interventions are discussed in detail elsewhere, ${ }^{51}$ we remind clinicians that powerful procoagulant agents carry a risk of causing thrombosis and, in patients with normal renal function, the anticoagulant effect of these new oral agents will dissipate quickly.

Hemodialysis should be considered for dabigatran-associated bleeding, since it is only $35 \%$ bound to plasma proteins. ${ }^{52}$ However, dialysis is not a suitable option for removing rivaroxaban or apixaban given their high degree of protein binding $(>95 \%)^{6}$

\section{Other Novel Oral Anticoagulants under Clinical Investigation}

The vast majority of the new oral anticoagulants in clinical development are direct FXa inhibitors, many of them with completed phase II clinical trials for prevention of VTE in the orthopedics surgery setting, and one of them (darexaban) in the ACS treatment and AF cardioembolic prevention scenarios. However, the development of darexaban was discontinued in September 2011 (-Table 4). In addition to FXa and direct thrombin inhibitors, factor IXa and factor $\mathrm{XIa}^{53}$ are among the suitable targets under current clinical investigation. ${ }^{54}$

\section{Conclusion}

Three new anticoagulants have now become available for prophylaxis and treatment of acute VTE and the prevention of cardioembolism in patients with AF. For these indications, these agents have demonstrated efficacy and safety that are comparable to more traditional alternatives, such as LMWH or well-controlled warfarin. The inability to easily measure the anticoagulant effect of the novel agents and the lack of an available antidote or evidence-based reversal strategy are 
disadvantages that will no doubt be addressed in future research. In some instances, the cost of these newer agents will likely be a barrier to their widespread use.

\section{Acknowledgments}

Dr. Rojas-Hernandez reports no relevant conflict of interest. Dr. Garcia has chaired or served advisory boards for Bayer, Boehringer Ingelheim, Bristol Meyers Squibb, CSL Behring, Daiich Sankyo, Ortho McNeill Janssen, and Pfizer.

\section{References}

1 Pinto DJ, Orwat MJ, Koch S, et al. Discovery of 1-(4-methoxyphenyl)7-oxo-6-(4-(2-oxopiperidin-1-yl)phenyl)-4,5,6,7-tetrahydro-1Hpyrazolo[3,4-c]pyridine-3-carboxamide (apixaban, BMS-562247), a highly potent, selective, efficacious, and orally bioavailable inhibitor of blood coagulation factor Xa. J Med Chem 2007;50(22):53395356

2 Wong PC, Jiang X. Apixaban, a direct factor Xa inhibitor, inhibits tissue-factor induced human platelet aggregation in vitro: comparison with direct inhibitors of factor VIIa, XIa and thrombin. Thromb Haemost 2010;104(2):302-310

3 Raghavan N, Frost CE, Yu Z, et al. Apixaban metabolism and pharmacokinetics after oral administration to humans. Drug Metab Dispos 2009;37(1):74-81

4 Bristol-Myers Squibb/Pfizer. Eliquis ${ }^{\circledR}$ (INN - apixaban) Summary of Product Characteristics. http://ec.europa.eu/health/documents/ community-register/2011/20110518102349/anx_102349_en.pdf

5 Wang L, He K, Maxwell B, et al. Tissue distribution and elimination of [14C]apixaban in rats. Drug Metab Dispos 2011;39(2):256-264

6 Kubitza D, Becka M, Voith B, Zuehlsdorf M, Wensing G. Safety, pharmacodynamics, and pharmacokinetics of single doses of BAY 59-7939, an oral, direct factor Xa inhibitor. Clin Pharmacol Ther 2005;78(4):412-421

7 Bayer Pharma AG. Xarelto ${ }^{\circledR}$ (INN - rivaroxaban) Summary of Product Characteristics. Available at: http://www.ema.europa. eu/docs/en_GB/document_library/EPAR_-_Product_Information/ human/000944/WC500057108.pdf. Accessed: February 1, 2013

8 Lang D, Freudenberger C, Weinz C. In vitro metabolism of rivaroxaban, an oral, direct factor Xa inhibitor, in liver microsomes and hepatocytes of rats, dogs, and humans. Drug Metab Dispos 2009; 37(5):1046-1055

9 Gnoth MJ, Buetehorn U, Muenster U, Schwarz T, Sandmann S. In vitro and in vivo P-glycoprotein transport characteristics of rivaroxaban. J Pharmacol Exp Ther 2011;338(1):372-380

10 Stangier J, Rathgen K, Stähle H, Gansser D, Roth W. The pharmacokinetics, pharmacodynamics and tolerability of dabigatran etexilate, a new oral direct thrombin inhibitor, in healthy male subjects. Br J Clin Pharmacol 2007;64(3):292-303

11 Blech S, Ebner T, Ludwig-Schwellinger E, Stangier J, Roth W. The metabolism and disposition of the oral direct thrombin inhibitor, dabigatran, in humans. Drug Metab Dispos 2008;36(2):386-399

12 Boehringer Ingelheim International GmbH. Pradaxa ${ }^{\circledR}$ (INN - dabigatran etexilate) Summary of Product Characteristics. Available at: http://www.ema.europa.eu/docs/en_GB/document_library/ EPAR_-_Product_Information/human/000829/WC500041059.pdf. Accessed: February 1, 2013

13 Härtter S, Koenen-Bergmann M, Sharma A, et al. Decrease in the oral bioavailability of dabigatran etexilate after co-medication with rifampicin. Br J Clin Pharmacol 2012;74(3):490-500

14 Boerhinger-Ingelheim. Dabigatran Advisory Committee Briefing Document. Available at: http://www.fda.gov/downloads/advisory committees/committeesmeetingmaterials/drugs/cardiovascular andrenaldrugsadvisorycommittee/ucm226009.pdf. Accessed: February 1, 2013
15 Lassen MR, Raskob GE, Gallus A, Pineo G, Chen D, Portman RJ. Apixaban or enoxaparin for thromboprophylaxis after knee replacement. N Engl J Med 2009;361(6):594-604

16 Lassen MR, Raskob GE, Gallus A, Pineo G, Chen D, Hornick P; ADVANCE-2 investigators. Apixaban versus enoxaparin for thromboprophylaxis after knee replacement (ADVANCE-2): a randomised double-blind trial. Lancet 2010;375(9717):807-815

17 Lassen MR, Gallus A, Raskob GE, Pineo G, Chen D, Ramirez LM; ADVANCE-3 Investigators. Apixaban versus enoxaparin for thromboprophylaxis after hip replacement. N Engl J Med 2010; 363(26):2487-2498

18 Goldhaber SZ, Leizorovicz A, Kakkar AK, et al. ADOPT Trial Investigators. Apixaban versus enoxaparin for thromboprophylaxis in medically ill patients. N Engl J Med 2011;365(23):2167-2177

19 Buller H, Deitchman D, Prins M, Segers A; Botticelli Investigators, Writing Committee. Efficacy and safety of the oral direct factor Xa inhibitor apixaban for symptomatic deep vein thrombosis. The Botticelli DVT dose-ranging study. J Thromb Haemost 2008; 6(8):1313-1318

20 Alexander JH, Becker RC, Bhatt DL, et al. APPRAISE Steering Committee and Investigators. Apixaban, an oral, direct, selective factor Xa inhibitor, in combination with antiplatelet therapy after acute coronary syndrome: results of the Apixaban for Prevention of Acute Ischemic and Safety Events (APPRAISE) trial. Circulation 2009;119(22):2877-2885

21 Alexander JH, Lopes RD, James S, et al. APPRAISE-2 Investigators. Apixaban with antiplatelet therapy after acute coronary syndrome. N Engl J Med 2011;365(8):699-708

22 Granger CB, Alexander JH, McMurray JJ, et al. ARISTOTLE Committees and Investigators. Apixaban versus warfarin in patients with atrial fibrillation. N Engl J Med 2011;365(11):981-992

23 Eikelboom JW, O'Donnell M, Yusuf S, et al. Rationale and design of AVERROES: apixaban versus acetylsalicylic acid to prevent stroke in atrial fibrillation patients who have failed or are unsuitable for vitamin K antagonist treatment. Am Heart J 2010;159(3):348, e1

24 Eriksson BI, Borris LC, Friedman RJ, et al. RECORD1 Study Group. Rivaroxaban versus enoxaparin for thromboprophylaxis after hip arthroplasty. N Engl J Med 2008;358(26):2765-2775

25 Kakkar AK, Brenner B, Dahl OE, et al. RECORD2 Investigators. Extended duration rivaroxaban versus short-term enoxaparin for the prevention of venous thromboembolism after total hip arthroplasty: a double-blind, randomised controlled trial. Lancet 2008;372(9632):31-39

26 Lassen MR, Ageno W, Borris LC, et al. RECORD3 Investigators. Rivaroxaban versus enoxaparin for thromboprophylaxis after total knee arthroplasty. N Engl J Med 2008;358(26):2776-2786

27 Turpie AG, Lassen MR, Davidson BL, et al. RECORD4 Investigators. Rivaroxaban versus enoxaparin for thromboprophylaxis after total knee arthroplasty (RECORD4): a randomised trial. Lancet 2009; 373(9676):1673-1680

28 Turpie AG, Lassen MR, Eriksson BI, et al. Rivaroxaban for the prevention of venous thromboembolism after hip or knee arthroplasty. Pooled analysis of four studies. Thromb Haemost 2011; 105(3):444-453

29 Cohen AT, Spiro TE, Büller HR, et al. Extended-duration rivaroxaban thromboprophylaxis in acutely ill medical patients: MAGELLAN study protocol. J Thromb Thrombolysis 2011;31(4): 407-416

30 Cohen AT, Buller HR, Mebazaa A, et al. Rivaroxaban compared with enoxaparin for the prevention of venous thromboembolism in acutely ill medical patients. The Annual Scientific Session of the American College of Cardiology. New Orleans, LA, 2011

31 Bauersachs R, Berkowitz SD, Brenner B, et al. EINSTEIN Investigators. Oral rivaroxaban for symptomatic venous thromboembolism. N Engl J Med 2010;363(26):2499-2510

32 Büller HR, Prins MH, Lensin AW, et al. EINSTEIN-PE Investigators. Oral rivaroxaban for the treatment of symptomatic pulmonary embolism. N Engl J Med 2012;366(14):1287-1297 
33 Mega JL, Braunwald E, Wiviott SD, et al. ATLAS ACS 2-TIMI 51 Investigators. Rivaroxaban in patients with a recent acute coronary syndrome. N Engl J Med 2012;366(1):9-19

34 Patel MR, Mahaffey KW, Garg J, et al. ROCKET AF Investigators. Rivaroxaban versus warfarin in nonvalvular atrial fibrillation. N Engl J Med 2011;365(10):883-891

35 Eriksson BI, Dahl OE, Rosencher N, et al. RE-NOVATE Study Group. Dabigatran etexilate versus enoxaparin for prevention of venous thromboembolism after total hip replacement: a randomised, double-blind, non-inferiority trial. Lancet 2007;370(9591):949-956

36 Eriksson BI, Dahl OE, Rosencher N, et al. RE-MODEL Study Group. Oral dabigatran etexilate vs. subcutaneous enoxaparin for the prevention of venous thromboembolism after total knee replacement: the RE-MODEL randomized trial. J Thromb Haemost 2007; 5(11):2178-2185

37 Ginsberg JS, Davidson BL, Comp PC, et al. RE-MOBILIZE Writing Committee. Oral thrombin inhibitor dabigatran etexilate vs North American enoxaparin regimen for prevention of venous thromboembolism after knee arthroplasty surgery. J Arthroplasty 2009; 24(1):1-9

38 Schulman S, Kearon C, Kakkar AK, et al. RE-COVER Study Group. Dabigatran versus warfarin in the treatment of acute venous thromboembolism. N Engl J Med 2009;361(24):2342-2352

39 Schulman S, Baanstra D, Eriksson H, et al. Dabigatran versus Placebo for Extended Maintenance Therapy of Venous Thromboembolism. ISTH, Kyoto, Japan; 2011

40 Schulman S, Kakkar AK, Schellong SM, et al. A randomized trial of dabigatran versus warfarin in the treatment of acute venous thromboembolism (RE-COVER II). The American Society of Hematology 2011 Annual Meeting. San Diego, CA, 2011

41 Schulman S, Eriksson H, Goldhaber SZ, et al. Dabigatran or Warfarin for Extended Maintenance Therapy of Venous Thromboembolism. ISTH, Kyoto, Japan; 2011

42 Oldgren J, Budaj A, Granger CB, et al. RE-DEEM Investigators. Dabigatran vs. placebo in patients with acute coronary syndromes on dual antiplatelet therapy: a randomized, double-blind, phase II trial. Eur Heart J 2011;32(22):2781-2789

43 Connolly SJ, Ezekowitz MD, Yusuf S, et al. RE-LY Steering Committee and Investigators. Dabigatran versus warfarin in patients with atrial fibrillation. N Engl J Med 2009;361(12):1139-1151
44 Eikelboom JW, Wallentin L, Connolly SJ, et al. Risk of bleeding with 2 doses of dabigatran compared with warfarin in older and younger patients with atrial fibrillation: an analysis of the randomized evaluation of long-term anticoagulant therapy (RE-LY) trial. Circulation 2011;123(21):2363-2372

45 Beasley BN, Unger EF, Temple R. Anticoagulant options-why the FDA approved a higher but not a lower dose of dabigatran. N Engl J Med 2011;364(19):1788-1790

46 Adam SS, McDuffie JR, Ortel TL, Williams JW Jr. Comparative effectiveness of warfarin and new oral anticoagulants for the management of atrial fibrillation and venous thromboembolism: a systematic review. Ann Intern Med 2012

47 Samama MM, Martinoli JL, LeFlem L, et al. Assessment of laboratory assays to measure rivaroxaban-an oral, direct factor Xa inhibitor. Thromb Haemost 2010;103(4):815-825

48 Lindahl TL, Baghaei F, Blixter IF, et al. Expert group on coagulation of the external quality assurance in laboratory medicine in Sweden. Effects of the oral, direct thrombin inhibitor dabigatran on five common coagulation assays. Thromb Haemost 2011; 105(2):371-378

49 Avecilla ST, Ferrell C, Chandler WL, Reyes M. Plasma-diluted thrombin time to measure dabigatran concentrations during dabigatran etexilate therapy. Am J Clin Pathol 2012;137(4): $572-574$

50 Stangier J, Feuring M. Using the HEMOCLOT direct thrombin inhibitor assay to determine plasma concentrations of dabigatran. Blood Coagul Fibrinolysis 2012;23(2):138-143

51 Kaatz S, Kouides PA, Garcia DA, et al. Guidance on the emergent reversal of oral thrombin and factor Xa inhibitors. Am J Hematol 2012;87(Suppl 1):S141-S145

52 Ganetsky M, Babu KM, Salhanick SD, Brown RS, Boyer EW. Dabigatran: review of pharmacology and management of bleeding complications of this novel oral anticoagulant. J Med Toxicol 2011;7(4):281-287

53 Al-Horani RA, Ponnusamy P, Mehta AY, Gailani D, Desai UR. Sulfated Pentagalloylglucoside Is a Potent, Allosteric, and Selective Inhibitor of Factor XIa. J Med Chem 2013 (e-pub ahead of print)

54 Ahrens I, Peter K, Lip GY, Bode C. Development and clinical applications of novel oral anticoagulants. Part II. Drugs under clinical investigation. Discov Med 2012;13(73):445-450 